\title{
Sistema inteligente para apoio à atualização espacial de ambientes reais para usuários que são cegos
}

\author{
João M. L. Brezolin ${ }^{1}$, Sandro Rama Fiorini ${ }^{2}$, Marcia de Borba Campos ${ }^{2}$, Rafael H. Bordini' ${ }^{2}$ \\ ${ }^{1}$ Instituto Federal Sul-Riograndense (IFSUL) - Campus Passo Fundo - RS \\ ${ }^{2}$ Pontifícia Universidade Católica do Rio Grande do Sul (PUCRS) - Porto Alegre - RS \\ joao.brezolin@passofundo.ifsul.edu.br, srfiorini@gmail.com, \\ marciabcampos@hotmail.com, rafael.bordini@pucrs.br
}

\begin{abstract}
The orientation and mobility skills are built since our birth. When we interact with an environment, we learn to place ourselves in space and guide us in relation to objects. However, many people with visual disabilities face spatial orientation problems in navigation in unfamiliar environments. This article describes an intelligent system that aims to capture recognize and transmit information about objects that are in the same space as its user. We believe that this system can help blind people to identify objects and understand spaces with more independence and autonomy.
\end{abstract}

Resumo. As habilidades de orientação e mobilidade são construídas desde o nosso nascimento. Quando interagimos com um ambiente, aprendemos a nos situar no espaço e a nos orientar em relação aos objetos. Entretanto, muitas pessoas com deficiência visual enfrentam problemas de orientação espacial na navegação em ambientes que não lhes são familiares. Este artigo descreve um sistema inteligente que objetiva captar, reconhecer e transmitir informações sobre objetos, que estão no mesmo espaço que seu utilizador. Acreditamos que este sistema possa auxiliar pessoas cegas a identificar objetos e a compreender espaços com mais independência e autonomia.

\section{Introdução}

Quando chegamos a novos lugares, nos valemos da visão para reconhecer onde estamos e o que está ao nosso redor. Através do sentido da visão, conseguimos classificar objetos avaliando características como forma, cor, peso e tamanho, localização e distância. Por meio da visão, podemos formar conceitos sobre os objetos como um todo, de forma que o recorte dessas características perfaz nossa percepção do ambiente. Assim, quem enxerga, constrói uma imagem visual da situação em que está e aprende sobre o que está ao seu redor pela observação. Esta aprendizagem, baseada na observação, é limitada para pessoas que são cegas, que se relacionam com o ambiente utilizando outros canais sensoriais e requerem apoio para ter uma leitura completa do ambiente.

A orientação espacial é estática, no que se refere à percepção das divisões físicas que formam um ambiente, mas também é dinâmica porque se refaz a cada nova informação que surge no ambiente. Estabelecer e manter a orientação espacial envolve um ciclo contínuo, que pode ser denominado de percepção-ação-imersão, no qual as ações são 
VII Congresso Brasileiro de Informática na Educação (CBIE 2018)

Anais do XXIX Simpósio Brasileiro de Informática na Educação (SBIE 2018)

guiadas pelas expectativas da pessoa que é cega em relação às informações perceptuais extraídas do ambiente, enquanto ela está imersa no mesmo.

O conhecimento espacial demanda presença espacial e imersão [Riecke and Von der Heyde 2002], e o processo de saber onde estamos quando nos deslocamos pelo espaço, e poder atualizar constantemente a nossa representação egocêntrica mental do ambiente, combinando com o nosso movimento, é denominado de "atualização espacial" [Riecke and Bülthoff 2004]. Nesse processo, as informações coletadas são confrontadas com conhecimentos prévios para que seja planejada uma estratégia de deslocamento. O ciclo de percepção-ação-imersão estabelece um processo de formação de conceitos, resultando na construção cognitiva de um mapa mental, que representa o ambiente no qual a pessoa que é cega se encontra [Golledge 1999, Carney et al. 2003].

Acreditando que um sistema de apoio à navegação de pessoas cegas pode prover recursos para ajudá-las na construção de seu mapa mental, e a construir o ciclo de percepção-ação-imersão, foi desenvolvido um sistema inteligente, apresentado neste artigo. Esse sistema é capaz de captar, reconhecer e transmitir informações sobre objetos do local onde o usuário se encontra. Para preservar a facilidade de uso e a robustez do sistema, sua arquitetura contém dois módulos. O Módulo do Usuário é responsável por realizar a captura das imagens dos objetos por meio de um dispositivo móvel e transmitir as informações resultantes do processo de classificação, de forma acessível, para o usuário que é cego. O Módulo do Agente Inteligente é responsável pelo processo de categorização de objetos identificados no ambiente. A conexão entre os módulos foi realizada utilizando o framework ROS (Robot Operational System) [Quigley et al. 2009]. O sistema inteligente foi desenvolvido utilizando o framework Jason [Bordini et al. 2007], que permite a criação de agentes capazes de implementar o processo de raciocínio necessário para classificar objetos e disponibilizar as informações de forma intuitiva para o usuário cego.

Este artigo está organizado em 6 seções. Uma breve fundamentação teórica é apresentada na Seção 2 e na Seção 3. O sistema desenvolvido é apresentado na Seção 4 e a avaliação do mesmo está na Seção 5. A Seção 6 apresenta conclusões da pesquisa.

\section{Mapas mentais, orientação e mobilidade}

As habilidades de orientação e mobilidade (O\&M) incluem capacidades motoras, cognitivas, afetivas e sociais, que permitem a pessoa conhecer, se relacionar e se deslocar em espaços e experimentar situações, que são apresentadas nesses ambientes. Conhecer o ambiente ao seu redor, e dominar técnicas de O\&M, significa poder se deslocar em um espaço com segurança e eficiência.

Para planejar seu deslocamento, as pessoas contam com um conjunto de recursos visuais que permitem estabelecer sua locomoção até o destino desejado [Golledge 1999]. A identificação de pontos de referência e obstáculos são exemplos de informações extraídas do ambiente pelo sujeito, no intuito de estabelecer sua orientação espacial. Nesse sentido, se estabelece um processo no qual os estímulos são organizados e confrontados com informações já armazenadas, resultando na transformação das sensações em significados. A orientação espacial pode ser definida como "o conhecimento do sujeito sobre sua distância e direção em relação às coisas observadas ou lembradas, e sua capacidade de manter estas relações espaciais à medida que se locomove" [Blasch et al. 1997]. Nesse 
VII Congresso Brasileiro de Informática na Educação (CBIE 2018)

Anais do XXIX Simpósio Brasileiro de Informática na Educação (SBIE 2018)

processo, dois aspectos são relevantes: a atualização espacial e o uso de quadros de referência. O primeiro diz respeito à capacidade da pessoa de manter informações sobre distâncias e direções de objetos à medida que se move. Essa característica compreende tanto a identificação de objetos simples, cuja a posição se modifica à medida que o sujeito se movimenta, quanto a identificação de objetos complexos, que podem assinalar a transição entre locais. Os quadros de referência podem ser classificados como egocêntricos ou alocêntricos. Os quadros egocêntricos assinalam informações que são recuperadas exclusivamente em relação à perspectiva da pessoa. Os quadros alocêntricos se referem ao processo no qual a pessoa relaciona objetos e locais independentemente do seu ponto de vista. Identificar objetos, a partir da localização de outros objetos, ou determinar locais que se localizam entre outros locais, são exemplos de operações inerentes ao uso de quadros de referência.

O termo "mapa mental" também é utilizado para designar formas utilizadas pela pessoa para recuperar imagens mentais sobre distâncias e direções de locais que estão fora de seu alcance perceptual [Golledge 1999]. Além de fazer uso de representações mentais do espaço, a pessoa pode reformular suas ações à medida que novas informações são adquiridas. Desta forma, as pessoas que são cegas têm capacidade para desenvolver habilidades motoras e mentais de forma que possam se movimentar em espaços que não sejam previamente conhecidos, desde que lhes sejam oferecidas informações e oportunidades para vivenciar esses espaços e para conhecer as características dos objetos.

\section{Espaços Conceituais e o ciclo de raciocínio de agentes BDI}

Agentes racionais são definidos como entidades ativas, capazes de analisar e atuar sobre o ambiente em que estão inseridos. As ações executadas pelo agente são resultantes do seu processo de raciocínio. O framework Jason [Bordini et al. 2007] foi desenvolvido com base em uma versão estendida da linguagem AgentSpeak, e permite especificar agentes BDI (Beliefs, Desires, Intentions), a partir da definição de sua base de crenças e planos. No agente Jason, as crenças são representadas por predicados de primeira ordem, que são denominados "literais de crença". O raciocínio do agente Jason ocorre em nível simbólico, a partir de operações lógicas realizadas com base em suas crenças. A implementação do nível de representação conceitual visa fundamentar as crenças do agente no que tange à classificação dos objetos identificados no ambiente. Para auxiliar nesse processo, foi implementado um nível de representação conceitual baseado na Teoria dos Espaços Conceituais [Gärdenfors 2000]. Esse nível foi incorporado ao ciclo de raciocínio do agente Jason para estabelecer fundamentação às crenças do agente, no que tange à classificação dos objetos identificados no ambiente.

Os espaços conceituais permitem representar conceitos e relações de similaridade em um espaço geométrico multidimensional. Esse espaço é decomposto em regiões, que formam domínios (cor, tamanho, forma, posição, etc.). As cores azul e vermelho, por exemplo, tendem a formar regiões convexas disjuntas do domínio de cor. Nesse espaço, conceitos são representados por conjuntos de regiões, que representam as características observadas em determinado objeto. A ideia dos espaços conceituais é fortemente influenciada pela Teoria dos Protótipos [Rosch 1999]. Nos espaços conceituais, o processo de classificação dos objetos compreende estabelecer a projeção das percepções do agente em regiões do espaço conceitual compondo um vetor de pontos. Este vetor deve, então, ser comparado por meio de uma métrica (como a distância euclidiana, por exemplo) com 
VII Congresso Brasileiro de Informática na Educação (CBIE 2018)

Anais do XXIX Simpósio Brasileiro de Informática na Educação (SBIE 2018)

o elemento mais significativo dos conceitos representados no espaço. Quanto menor a distância, maior a possibilidade desta percepção representar a instância de um conceito representado no espaço conceitual. Esse processo permite estabelecer um grau de similaridade entre a observação e os elementos prototípicos do espaço conceitual.

Esse processo de classificação dos objetos foi acoplado ao ciclo de raciocínio do agente Jason para que ele classifique os objetos, que são observados no local em que o usuário se encontra. $\mathrm{O}$ agente também busca classificar o ambiente no qual o usuário se encontra com base no total de objetos identificados. Assim, ao final do processo, o usuário também recebe essa informação, que pode auxiliar na construção cognitiva alocêntrica do ambiente.

\section{Sistema Inteligente de apoio às pessoas com deficiência visual}

Existem diferentes sistemas interativos para apoiar pessoas cegas a explorar espaços abertos ou fechados, familiares ou desconhecidos, e a se locomover por esses com certa segurança. Há sistema que fornecem descrições verbais sobre o ambiente, sem que o usuário esteja nesse [de Borba Campos et al. 2015] ou que fornecem informações no momento em que o usuário se encontra em um determinado espaço por meio de GPS [Morad 2010]. Mas, diferente de muitos desses, o sistema aqui apresentado tem como propósito servir para a construção do espaço, a partir da relação da pessoa com os objetos. Dentre as possibilidades de construção do espaço pela pessoa cega, a partir do uso do sistema, citam-se:

- Espaço corporal: utilizando o seu próprio corpo como referência, o usuário cego pode localizar objetos a partir de relações entre si e esses objetos.

- Espaço de ação: a partir da indicação do que há ao seu redor, o usuário cego pode construir o espaço representativo em contato com o objeto.

- Espaço dos objetos: trata da posição dos objetos, a partir do espaço corporal perceptivo. Passa-se, então, à construção do espaço representativo, que ocorre quando o usuário cego não precisa ter contato com os objetos para internalizar a posição desses.

- Espaço geométrico: a partir de um sistema de coordenação, o usuário cego passa a elaboração de mapas mentais.

- Espaço abstrato: se refere à capacidade de manejo dos conceitos para elaboração de rotas, mapas e outros.

Desta forma, a familiarização de ambientes é um exemplo de como o sistema pode ser utilizado porque oferece ao usuário cego condições de obter informações sobre um espaço familiar ou desconhecido. Assim, o usuário pode se posicionar na porta de entrada de uma sala, por exemplo, e, a partir do sistema, identificar um objeto, percorrer o ambiente até esse objeto e retornar ao ponto inicial (porta de entrada). Poderá, progressivamente, incluir todos os objetos da sala e ampliar a exploração do espaço. Ao final, o usuário deverá ser capaz de relacionar todos os objetos do ambiente em relação à sua posição inicial, que era a porta, construindo um mapa mental do cenário.

O sistema está organizado em dois módulos. O Módulo do Usuário compreende um aplicativo instalado em um dispositivo móvel, com o qual o usuário que é cego interage. O aplicativo é responsável pela captura de imagens e, posteriormente, pela transmissão das informações para o usuário. O Módulo do Agente Inteligente é responsável pelo processo de identificação e classificação dos objetos, a partir de imagens enviadas 
VII Congresso Brasileiro de Informática na Educação (CBIE 2018)

Anais do XXIX Simpósio Brasileiro de Informática na Educação (SBIE 2018)

pelo aplicativo do dispositivo móvel. A conexão entre o Módulo do Usuário e o Módulo do Agente Inteligente é realizada através do sistema operacional ROS, que envia as imagens capturadas pela câmara do smartphone, em nodos para serem lidos pelo Módulo do Agente Inteligente. Quando o aplicativo é executado pelo usuário, automaticamente é realizada uma conexão com o servidor ROS que, quando estabelecida, o aplicativo libera o seu uso e inicia o processo de captura de imagens. O usuário direciona a câmera do seu dispositivo para o ponto que deseja obter informações e permanece na mesma direção até que o aplicativo indique que uma imagem foi capturada. Caso o usuário necessite realizar, ou reiniciar o processo de reconhecimento de objetos, basta que redirecione a câmera do smartphone ao local desejado e o processo de classificação é reestabelecido. Cada imagem capturada é transferida para o servidor para que ocorra o processo de classificação, e, novamente, ao usuário com informações sobre os objetos identificados. A interface sonora é realizada por meio de um recurso implementado por meio do Text-to-Speech (Figura 1).

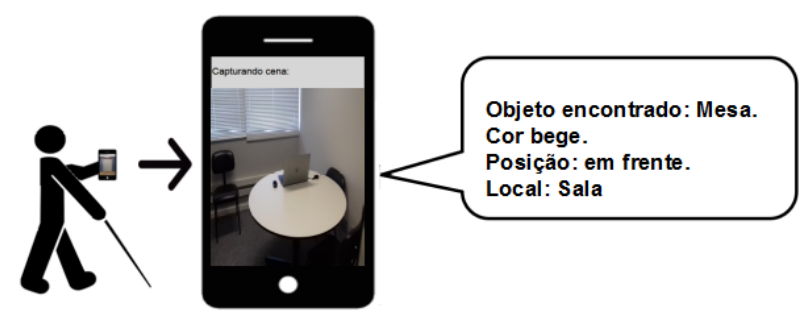

Figura 1. Interação do usuário com o aplicativo

O processo de identificação e classificação dos objetos tem início com a segmentação da imagem capturada. Para tal, foi utilizada a biblioteca gráfica OpenCV [Huaman et al. 2018] (Figura 2). O processo é assim estabelecido: inicialmente, é extraído um conjunto de contornos, que delimita os objetos encontrados na imagem. Para cada contorno, se estabelece um processo de extração de características. Extrai-se, de cada objeto, informações sobre sua posição egocêntrica, cor predominante e forma. A posição do objeto é estabelecida a partir de um ponto central da cena capturada, e a localização do objeto é estabelecida em relação a esse ponto. A cor predominante e a forma são determinadas a partir da projeção de informações extraídas em domínios do espaço conceitual. O domino da cor é especificado a partir do modelo de cores RGB, que é o padrão utilizado pela biblioteca OpenCV. O domínio da forma é especificado a partir de Hu-Moments [Hu 1962]. O conjunto dessas informações permite compor um vetor de características, que possibilita identificar o objeto capturado.

O vetor de características é enviado ao agente Jason [Bordini et al. 2007], que inicia o processo de classificação. Antes de enviar as informações para o nível simbólico, o agente realiza um processo de classificação, a partir da projeção das informações recebidas no espaço conceitual. Para realizar a especificação do nível conceitual é utilizada a linguagem CSML (Conceptual Space Markup Language), que permite representar elementos do espaço conceitual com base na álgebra proposta por [Adams and Raubal 2009]. A API CSML foi integrada ao ciclo de raciocínio do agente para possibilitar a criação do nível conceitual e implementar os cálculos de similaridade entre as observações e os elementos prototípicos de objetos especificados no espaço conceitual. 
VII Congresso Brasileiro de Informática na Educação (CBIE 2018)

Anais do XXIX Simpósio Brasileiro de Informática na Educação (SBIE 2018)



Figura 2. Processo de classificação de objetos

Os dados extraídos de cada contorno são utilizados para especificar uma instância temporária, que precisa ser associada a um conjunto de regiões (que representam conceitos) do espaço conceitual. Calcula-se a distância entre o vetor de pontos da instância e os vetores de pontos dos conceitos candidatos, ou seja, conceitos que poderão ser associados ao objeto, permitindo classificá-lo. O conceito cujo centroide está mais próximo dos pontos especificados na instância é eleito para classificar o objeto observado, e passa a representar o objeto. Esta informação, associada a posição do objeto, compõe uma mensagem que é enviada ao nível simbólico de raciocínio do agente. Nesse, procedese com a adição desta crença, que representa o objeto na sua base de conhecimentos [Brezolin et al. 2015]. Uma vez concluído esse processo, o agente organiza e compõem as mensagens descritivas, que são enviadas ao usuário.

As mensagens são organizadas de acordo com a posição dos objetos na imagem. Foram criadas regiões no espaço conceitual, que especificam as posições esquerda, centro e direita, conforme apresentado na Figura 3.

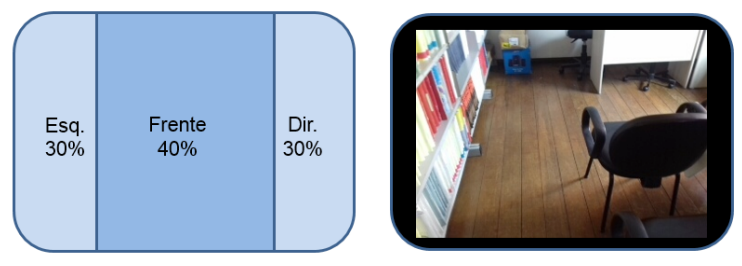

Figura 3. Definição da posição dos objetos na imagem

O agente inteligente inicia a narração dos objetos da esquerda para direita. Essa premissa busca facilitar a construção do mapa mental do ambiente realizada pelo usuário cego. No caso da Figura 3, por exemplo, a narração iniciará pela estante e encerrará pela cadeira. Caso algum desses objetos não possa ser classificado, o sistema emitirá a mensagem indicando que há um objeto não identificado, assinalando a sua posição. Já na Figura 1, há apenas um objeto identificado ao centro. Nesse caso, apenas a mensagem referente a este objeto é reproduzida. Ao final do processo, o agente inteligente avalia os objetos encontrados e informa ao usuário o ambiente em que, provavelmente, se encontram.

Diferente das pesquisas realizadas por [Mombach and Welfer 2013] e 
VII Congresso Brasileiro de Informática na Educação (CBIE 2018)

Anais do XXIX Simpósio Brasileiro de Informática na Educação (SBIE 2018)

[Serrão 2015], optamos por implementar um agente inteligente que realiza o processo de classificação com base no processo de raciocínio do agente. Ou seja, o agente infere sobre um conjunto de características e não sobre os valores dos pixels que compõem a imagem. Foram utilizados descritores de cor e forma para realizar o processo de classificação de objetos, mas o sistema se mostra flexível para associar outros descritores como textura e tamanho, por exemplo.

\section{Avaliação do Sistema Inteligente de apoio às pessoas com deficiência visual}

Foram realizadas duas avaliações do sistema, cada uma com um usuário cego, em momentos e ambientes diferentes. Os usuários foram selecionados por conveniência. O objetivo foi verificar se o sistema auxiliava os usuários a (re)conhecerem ambientes e identificar objetos dispostos nesses. Após a concordância em participar (por meio do TCLE), foi feita uma entrevista para conhecer o perfil dos participantes e suas expectativas com relação ao uso do sistema. Após a realização do experimento, foi realizada outra entrevista para avaliar critérios de usabilidade e de satisfação de uso. As entrevistas e o experimento foram gravados em áudio e em vídeo. Cada experimento durou 30 minutos.

O Modulo do Usuário foi testado em um dispositivo Samsung Galaxy Gran Prime Duos com 8 GB de memória RAM e com sistema Operacional Android Lolipop 5.1. O Modulo do Agente Inteligente foi instalado em um servidor Ubuntu versão 14.04 em uma máquina virtual com 4 GB de RAM. O Host dessa máquina virtual é um Notebook Dell Inspiron 15 com processador iCore 7 e 16 GB de RAM.

\subsection{Experimento 1}

O participante $\mathrm{P} 1$ é cego de nascença, do gênero masculino, se encontra na faixa etária dos 18 a 25 anos, é aluno de curso superior na área da saúde em uma universidade privada. P1 tem experiência no uso de smartphones e faz uso do leitor de tela TalkBack, porém nunca utilizou aplicativos para auxiliá-lo a se deslocamento por ambientes abertos e fechados. Para realizar essa tarefa, geralmente faz uso da bengala e conta com o auxílio de outras pessoas.

Foi definido o local de teste, que não fosse de conhecimento de P1, mas que o avaliador pudesse ter liberdade para manejar as mobílias e os objetos, e não tivesse restrição de entrada. Foi escolhida uma sala que tivesse janela, estantes de diferentes tamanhos, mesa, cadeiras, notebook, mochila. O espaço foi preparado e foi feito o cadastramento dos objetos do ambiente para criar a base de conceitos.

Foram definidas três tarefas: (1) a partir da porta, reconhecer dois objetos quaisquer, (2) se deslocar até a mesa, (3) fazer um mapa tátil do cenário (Figura 4), realizadas com sucesso. Durante o experimento, ocorreu casos em que a leitura das mensagens não acompanhava a movimentação da câmara do celular. Também houve momentos em que o sistema classificou objetos com base em capturas parciais, ocasionando classificações equivocadas. O experimento motivou a adição do recurso de vibração para alertar o usuário sobre o recebimento de novas mensagens. P1 compreendeu o espaço e os objetos, identificando a mesa, cadeiras, mochila, e a estante maior, e a relação entre esses objetos com facilidade. Considerou que foi fácil utilizar o aplicativo e aprender como utilizá-lo. Porém, ressaltou que o aplicativo apresentou demora na reprodução das mensagens. P1 recomendaria o uso do aplicativo, mas sugeriu melhorar a interface sonora e as mensagens sobre o posicionamento dos objetos. 
VII Congresso Brasileiro de Informática na Educação (CBIE 2018)

Anais do XXIX Simpósio Brasileiro de Informática na Educação (SBIE 2018)

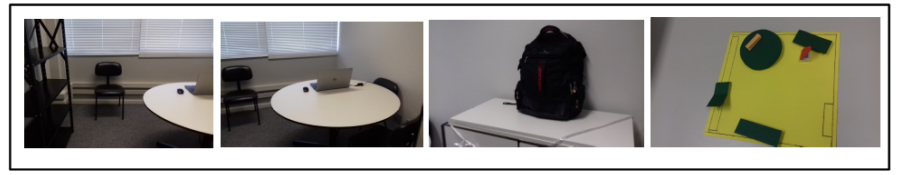

Figura 4. Imagem do sala e mapa tátil elaborado pelo usuário P1

\subsection{Experimento 2}

O participante P2 possui cegueira adquirida na adolescência e está na faixa etária dos 36 aos 45 anos. Tem experiência limitada com smartphones. Faz uso, principalmente, da bengala. Foi selecionada uma sala (Figura 5), de conhecimento de P2, que costuma usar o tato para reconhecer os objetos. Esta sala possui um corredor, armário de livros, cadeiras, mesa, notebook, janelas com persianas. Esses objetos foram cadastrados na base de conhecimento do sistema. Foram definidas 5 tarefas: (1) a partir da porta de entrada, reconhecer uma janela e um conjunto de cadeiras, (2) se deslocar até a janela, (3) retornar a porta de entrada, (4) se deslocar pelo corretor formado pelo armário de livros e cadeiras, (5) identificar o objeto em cima da mesa (notebook), realizadas com sucesso. P2 também considerou o sistema de fácil utilização, apesar de ter tido dificuldades para compreender que o sistema informava a posição dos objetos em relação à posição da câmera e não em relação ao seu posicionamento corporal. Por isso, considera importante haver uma orientação prévia sobre como direcionar a câmera do smartphone. Considerou o uso da interface sonora e o recurso de vibração importantes para compreender a relação entre os objetos. P2 fez menos uso do tato para identificar os objetos. Recomendou o uso do sistema, e sugeriu que objetos menores pudessem ser reconhecidos.

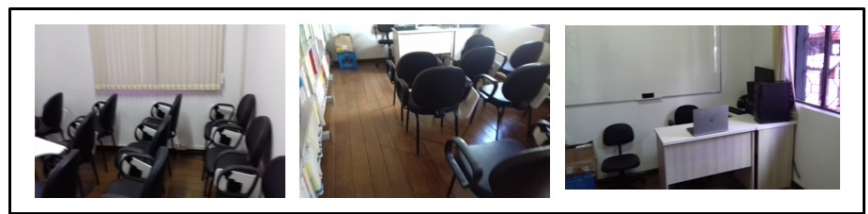

Figura 5. Imagem da sala do experimento 2

No uso do sistema, é comum o usuário direcionar a câmara do celular para diferentes lugares, que possuem imagens não cadastradas, e que até mesmo podem não ter significados relevantes. Considerando os dados do experimento 2 , o sistema adotado pode filtrar e eliminar $62 \%$ das imagens, que foram capturadas enquanto a câmara estava sendo movimentada. As demais imagens (38\%) refletem objetos reconhecidos e outros que não puderam ser classificados devido não estarem na base ou por terem sido capturados de forma parcial. Dos objetos corretamente capturados $83 \%$ foram classificados corretamente pela abordagem de espaços conceituais. Os resultados demonstraram que o sistema obteve um bom resultado com relação aos objetos classificados assim como permitiu confirmar a distância máxima para reconhecimento dos objetos [Brezolin et al. 2017].

Também se observou que a velocidade no processo de classificação influenciou significativamente na reprodução das mensagens realizada pelo smartphone. Nesse sentido, no comparativo realizado utilizado o software SURF (Speeded Up Robust Features), se verificou que o processo de classificação realizado pelo software foi mais lento do que 
VII Congresso Brasileiro de Informática na Educação (CBIE 2018)

Anais do XXIX Simpósio Brasileiro de Informática na Educação (SBIE 2018)

o realizado pela abordagem dos espaços conceituais. Esse fato ocasionou a reprodução de mais mensagens atrasadas para o usuário cego. A abordagem utilizada permitiu minimizar esse problema (não evitá-lo), pois o vetor de características é menor do que o utilizado pelo SURF e os cálculos são disponibilizados mais rapidamente, mesmo considerando a transmissão de dados nos três níveis de representação (Figura 2).

\section{Conclusão}

Este documento apresenta um sistema, direcionado a usuários cegos, que permite a compreensão de ambientes por meio do reconhecimento de objetos, que estão no espaço. A proposta se diferencia de outras, por realizar o reconhecimento de objetos com base no processo de raciocínio de um agente inteligente. Para tanto, foi implementado um nível de representação conceitual. Seguindo as proposições de Adams e Rubal [Adams and Raubal 2009], foram definidos os domínios de cor e forma. O domínio cor foi definido com base no modelo RGB, e a forma em Hu-moments. A partir da CSML API e o software matemático q-hull, foi possível definir as regiões e seus respectivos elementos prototípicos. Dessa forma, foi possível definir classificadores, que realizam a comparação de objetos observados no ambiente com os elementos prototípicos de cada conceito.

A avaliação foi realizada com dois usuários cegos, em diferentes situações de exploração de ambientes conhecidos e não conhecidos. Os resultados mostraram que o uso do sistema permitiu o reconhecimento dos ambientes e das relações entre os objetos. Indicaram, ainda, que, gradualmente, os usuários fizeram menos uso do tato para reconhecer os objetos. Nas entrevistas, os usuários fizeram sugestões de melhorias na interface sonora e na detecção de objetos menores. Também foram realizados estudos comparando a solução baseada em espaços conceituais com histogramas e com um software de reconhecimento de imagem, o SURF. Resultados indicaram que a proposta foi mais vantajosa em termos de reconhecimento de objetos e tempo de retorno das informações ao usuário.

Como trabalho futuro, se pretende melhorar a usabilidade e adicionar novas funcionalidades, por exemplo, disponibilizar um histórico de um processo de reconhecimento, melhorar as relações entre os objetos associados a um mesmo ambiente, melhorar a interface sonora, adicionar novos domínios ao espaço conceitual para tornar o processo de classificação mais preciso.

\section{Referências}

Adams, B. and Raubal, M. (2009). Conceptual space markup language (CSML): towards the cognitive semantic web. In Proceedings of the 3rd IEEE International Conference on Semantic Computing (ICSC 2009), pages 253-260.

Blasch, B. B., Welsh, L., R., and Wiener, W. R. (1997). Perception, locomotion, and orientation, volume 1. New York: AFB Press.

Bordini, R. H., Hübner, J. F., and Wooldridge, M. (2007). Programming Multi-Agent Systems in AgentSpeak Using Jason. John Wiley \& Sons.

Brezolin, J. M. L., Fiorini, S. R., de Borba Campos, M., and Bordini, R. H. (2015). Using conceptual spaces for object recognition in multi-agent systems. In PRIMA 2015: Principles and Practice of Multi-Agent Systems - 18th International Conference, Bertinoro, Italy, October 26-30, 2015, Proceedings, pages 697-705. 
VII Congresso Brasileiro de Informática na Educação (CBIE 2018)

Anais do XXIX Simpósio Brasileiro de Informática na Educação (SBIE 2018)

Brezolin, J. M. L., Fiorini, S. R., de Borba Campos, M., and Bordini, R. H. (2017). Use of conceptual representations based on conceptual spaces theory applied to BDI agents. In 2017 Brazilian Conference on Intelligent Systems, BRACIS 2017, Uberlândia, Brazil, October 2-5, 2017, pages 157-162.

Carney, S., Engbretson, C., Scammell, K., and Sheppard, V. (2003). Teaching students with visual impairments: A guide for the support team.

de Borba Campos, M., Sánchez, J., Damasio, J., and Inácio, T. (2015). Usability evaluation of a mobile navigation application for blind users. In Universal Access in Human-Computer Interaction. Access to the Human Environment and Culture - 9th International Conference, UAHCI 2015, Held as Part of HCI International 2015, Los Angeles, CA, USA, August 2-7, 2015 Proceedings, Part IV, pages 117-128.

Gärdenfors, P. (2000). Conceptual Spaces: The Geometry of Thought. MIT Press.

Golledge, R. G. (1999). Human wayfinding and cognitive maps. US: Johns Hopkins University Press.

Hu, M. K. (1962). Visual pattern recognition by moment invariants. vol. IT-8:179-187.

Huaman, A. et al. (2018). OpenCV Documentation. Disponível em: http://docs.opencv.org/2.4/doc/ tutorials/tutorials.html. Acessado em: 31 mai 2018.

Mombach, J. G. and Welfer, D. (2013). Proposta de um aplicativo móvel para percepção de imagens estáticas por alunos com deficiência visual. In In Anais do XXIV Simpósio Brasileiro de Informática na Educação (SBIE 2013)., Campinas, São Paulo.

Morad, A. H. (2010). Gps talking for blind people. In Journal of emerging technologies in Web Inteligence, VOL. 2, NO. 3.

Quigley, M., Conley, K., Gerkey, B. P., Faust, J., Foote, T., Leibs, J., Wheeler, R., and $\mathrm{Ng}$, A. Y. (2009). Ros: an open-source robot operating system. In Proceedings of the ICRA Workshop on Open Source Software.

Riecke, B. E. and Bülthoff, H. H. (2004). Spatial updating in real and virtual environments: Contribution and interaction of visual and vestibular cues. In Proceedings of the 1st Symposium on Applied Perception in Graphics and Visualization, APGV'04, pages 9-17.

Riecke, B. E. and Von der Heyde, M. (2002). Qualitative Modeling of Spatial Orientation Processes using Logical Propositions: Interconnecting Spatial Presence, Spatial Updating, Piloting, and Spatial Cognition. Max Planck Institute for Biological Cybernetics, Tübingen, Germany.

Rosch, E. (1999). Principles of categorization. MIT Press, Cambridge, Massachusetts.

Serrão, M. e. a. (2015). Computer vision and gis for the navigation of blind persons in buildings. Universal Access in the Information Society, 14(1):67-80. 